\title{
Anwendungsbeispiel zur quantitativen Abschätzung von Abflusssituation und Abflussminderung
}

7.1 Einführung - 160

7.2 Methoden -160

7.3 Ergebnisse - 163

7.4 Zusammenfassung - 170

Literatur - 171 


\subsection{Einführung}

Zur Illustration der vorgestellten Methoden wird die Verringerung des Abflussscheitels durch Aufweitung eines Grabens und Veränderung der Flächenbewirtschaftung durchgespielt. Gegenstand des Planungsbeispiels ist die Ortschaft Birnbach im südlichen Landkreis Regensburg, die in der Vergangenheit häufiger von kleineren Überschwemmungen betroffen war. Der Siedlungsbereich liegt im Kopfeinzugsgebiet des Allersdorfer Bachs, der bei Schierling in die Große Laaber mündet. Ursache der Überschwemmungen ist die Kessellage der Ortschaft (• Abb. 7.1, oben). Auf dem Weg von der Wasserscheide zum Allersdorfer Bach verlaufen die Entwässerungswege verschiedener Teileinzugsgebiete durch die südöstlichen bzw. nordwestlichen Ortsrandlagen. Das Einzugsgebiet ist rund $1,5 \mathrm{~km}^{2}$ groß und überwiegend landwirtschaftlich genutzt. Vor allem im Bereich westlich der Hauptverbindungsstraße, die in Nord-Süd-Richtung und durch die Ortschaft verläuft, beträgt der Ackeranteil fast $100 \%$.

Das Gebiet wird durch ein langes, verästeltes Grabensystem sehr feingliedrig erschlossen. Die Gräben sind parallel zu den Feldwegen angelegt, die überwiegend in den Tiefenlinien verlaufen (siehe das kleine, im Nordwesten gelegene, 47 ha große Teileinzugsgebiet in - Abb.7.1, unten). Die Gräben untergliedern das Teileinzugsgebiet in noch feinere Einzugsgebiete (A-D) und entwässern schließlich gemeinsam über einen in der Tiefenlinie verlaufenden Sammelgraben in den Allersdorfer Bach. Die Länge des künstlichen Grabensystems übersteigt die Länge des Allersdorfer Bachs um ein Vielfaches. Allein im nordwestlichen Teileinzugsgebiet ist das künstliche Grabensystem sechsfach länger als der zugehörige Abschnitt des Allersdorfer Bachs.

\subsection{Methoden}

Um die Abflusssituation im Gebiet quantitativ $\mathrm{zu}$ fassen, wird für das ausgewiesene Teileinzugsgebiet exemplarisch der Scheitel einer Abflusswelle ermittelt, der am Bezugspunkt im Graben vor der Ortschaft (vgl. - Abb. 7.1, unten) auftreten kann. Dazu wird zunächst der Anteil des Niederschlags bestimmt, der bei einem typischen Starkregen abfließt. Dann wird die Konzentrationszeit des Gebietes ermittelt, indem der Fließweg des Oberflächenabflusses von der Wasserscheide bis zum Bezugspunkt analysiert wird. Aus der Höhe des abflusswirksamen Niederschlags, der Einzugsgebietsgröße und der Konzentrationszeit ergibt sich der zu erwartende Scheitelabfluss.

In einem zweiten Schritt wird der Einfluss der Gräben auf die Abflusskonzentration und damit auf den Wellenscheitel untersucht, indem fiktiv von einer Aufweitung der Gräben und der Herstellung hydraulisch rauer Bedingungen ausgegangen wird, z. B. durch das Tolerieren eines dichten Bewuchses und den Verzicht auf eine regelmäßige Mahd. Der Effekt dieser Maßnahmen lässt sich abschätzen, indem die veränderte Konzentrationszeit und damit der veränderte Scheitelabfluss ermittelt und der aktuellen Situation gegenübergestellt wird. Und schließlich wird noch die Wirkung einer flächendeckenden Mulchdirektsaat betrachtet. Das Vorgehen entspricht dem Rechenweg typischer Bemessungs- und Dimensionierungsaufgaben und lässt sich auf viele Anwendungsfälle übertragen.

\subsubsection{Bemessungsniederschlag und abflusswirksame Niederschlagshöhe}

Die Bestimmung der abflusswirksamen Niederschlagshöhe mit dem CN-Verfahren (vgl. Kap.4) erfordert die Vorgabe eines Bemessungsniederschlags sowie Informationen zur Landnutzung und hydrologischen Bodengruppe (vgl. > Anhang 8.6).

Als Niederschlagsszenario wird ein Regen mit $51 \mathrm{~mm}$ in $1 \mathrm{~h}$ angenommen. Im Einzugsgebiet entspricht dies einem Starkregen mit einem Wiederkehrintervall von 100 Jahren nach 

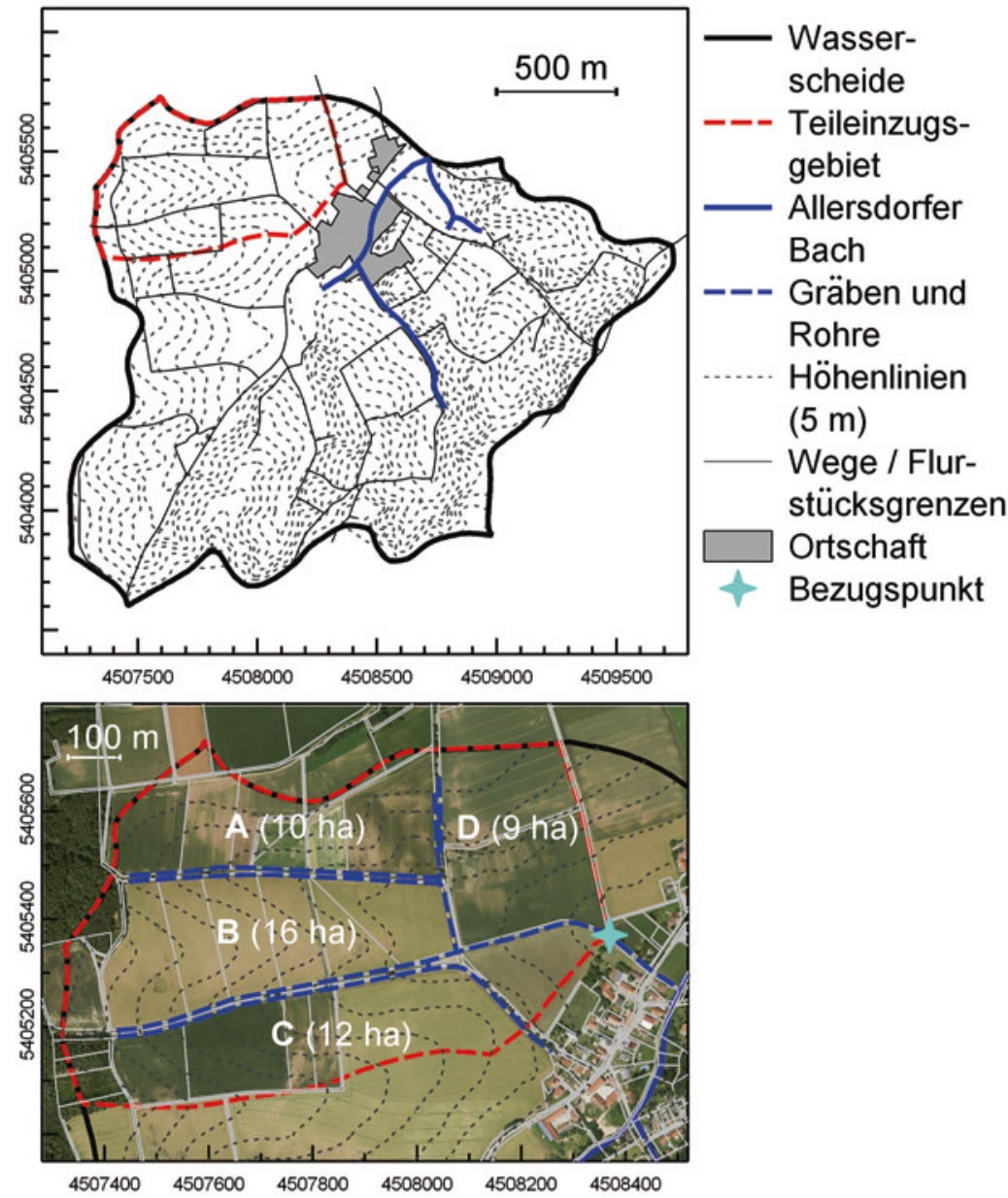

- Abb. 7.1 Übersichtskarte des Einzugsgebietes der Ortschaft Birnbach (oben). Im Gebiet sind fein verästelte Grabensysteme vorhanden, die exemplarisch für ein Teileinzugsgebiet dargestellt sind (unten). Durch die Gräben wird wiederum in vier weitere Teilflächen (A bis D) untergliedert. (Geobasisdaten: Bayerische Vermessungsverwaltung)

KOSTRA (zum Vergleich, ein 30-jährlicher, einstündiger Regen hätte eine Höhe von $41 \mathrm{~mm}$ ) [1]. Die Landnutzung wurde aus Luftbildern und einer Geländebegehung erhoben und mit einer Karte der hydrologischen Bodengruppe (für Bayern verfügbar über das Landesamt für Umwelt) in einem Geographischen Informationssystem verschnitten. Die Auswertung ergab, dass das Gebiet nahezu vollständig ackerbaulich genutzt wird (Grünland- und Waldanteil betragen jeweils etwa $5 \%$; der Anteil der Straßen an der Gesamtfläche beträgt $<2 \%$ ) und dass die Fläche $\mathrm{zu}$ über 2/3 der hydrologischen Bodengruppe C zugeordnet ist (nicht graphisch dargestellt). Die Bewirtschaftung erfolgte zum Zeitpunkt der Geländebegehung etwa zu gleichen Teilen in Hauptgefällerichtung wie parallel dazu.

Es wird von mittleren Feuchtebedingungen ausgegangen und vereinfachend angenommen, dass die hydrologische Bodengruppe $\mathrm{C}$ das Gebiet adäquat repräsentiert und dass die Gesamtfläche ackerbaulich genutzt wird. Für das Planungsbeispiel wird daher einheitlich ein $\mathrm{CN}$-Wert von 80 unterstellt. Das entspricht nach - Abb.4.3 einer 
mittleren Bodenbedeckung von $10 \%$ bzw. nach - Tab. 4.2 einem CN-Wert, der z. B. bei Sommergetreide im April und Anfang September und bei Mais Ende Mai und Anfang Oktober zu erwarten ist.

Für reale Planungsfälle sollte eine detailliertere Berechnung erfolgen, indem die existierenden Landnutzungsformen mit den hydrologischen Bodengruppen verschnitten und die Abflussbildung dann für jede resultierende Einheit individuell bestimmt wird. Die Dauerstufe des Niederschlags sollte zudem der Konzentrationszeit des Oberflächenabflusses im Einzugsgebiet entsprechen. Da die Konzentrationszeit auf Ackerflächen über das Jahr variiert, sollten ggf. Modellregen unterschiedlicher Dauerstufen (je nach Konzentrationszeit) und Jährlichkeiten betrachtet werden, um die Größenordnung typischer Abflussreaktionen einschätzen $\mathrm{zu}$ können. Hier wird aber vereinfachend nur ein einziger, mittlerer Fall betrachtet. - Tab. 7.1 enthält eine Zusammenfassung der Eingangsparameter für die Ermittlung der abflusswirksamen Niederschlagshöhe nach dem CN-Verfahren.

\subsubsection{Ermittlung der Konzentrationszeit}

Zur Abschätzung der Konzentrationszeit wird die Geschwindigkeitsmethode (vgl. Abschn. 5.3.3) verwendet. Dazu wird zunächst der längste Entwässerungsweg im Einzugsgebiet bestimmt und anhand von Abflusstyp, Gefälle und Fließpfadquerschnitt in hydraulisch ähnliche Abschnitte untergliedert. Im Beispielgebiet gliedert er sich in vier Abschnitte (- Abb. 7.2). Der erste (A1) beginnt an der Wasserscheide und erstreckt sich über die landwirtschaftliche Nutzfläche bis zum Hangfuß. Bei Starkregen ist hier schichtförmiger Abfluss und flacher, konzentrierter Abfluss in Rinnen und Rillen zu erwarten. Am Hangfuß tritt
- Tab. 7.1 Eingangsparameter zur Bestimmung der abflusswirksamen Niederschlagshöhe im nordwestlichen Teil des Einzugsgebietes des Allersdorfer Baches bei Birnbach (Landkreis Regensburg)

\begin{tabular}{|c|c|c|}
\hline Parameter & Einheit & Wert \\
\hline $\begin{array}{l}\text { Niederschlags- } \\
\text { höhe }(\mathrm{N})\end{array}$ & $\mathrm{mm}$ & 51 \\
\hline $\begin{array}{l}\text { Hydrologische } \\
\text { Bodengruppe }\end{array}$ & - & $\mathrm{C}$ \\
\hline CN-Wert & - & $\begin{array}{l}80 \text { (Getreide mit } \\
\text { hoher Abfluss- } \\
\text { neigung) }\end{array}$ \\
\hline
\end{tabular}

das Wasser in ein Grabensystem über, das das Wasser bis in den Allersdorfer Bach leitet (Abschnitte A2, A3 und A4). Über die hydraulischen Eigenschaften der einzelnen Fließpfade und die GMS-Gleichung lässt sich die Fließzeit des Oberflächenabflusses in den einzelnen Abschnitten des Fließpfades abschätzen. Die Summe dieser Fließzeiten ergibt die Konzentrationszeit des Gebietes. Mit dem gleichen Ansatz kann auch der Einfluss der Grabengestaltung auf die Abflusskonzentration ermittelt werden, indem Gerinnequerschnitt und Rauheit verändert und die Fließzeiten der einzelnen Abschnitte bei gleicher Abflussrate neu ermittelt werden.

Zur Parametrisierung der GMS-Gleichung werden Neigung und Länge der einzelnen Fließpfadabschnitte aus der topographischen Karte bzw. dem digitalen Höhenmodell ermittelt. Repräsentative Querprofile der einzelnen Grabenabschnitte wurden im Gelände erfasst (A2, A3 und A4 sind überwiegend als steile, geräumte Trapeze ausgebildet). Anhand der kartierten Oberflächenbeschaffenheit wurden Rauheitsbeiwerte für Schichtabfluss und den Abfluss in Gräben aus - Tab. 8.2 und - Tab.5.1 entnommen. - Tab. 7.2 enthält eine Zusammenfassung der hydraulischen Parameter der genannten Fließpfadabschnitte. 


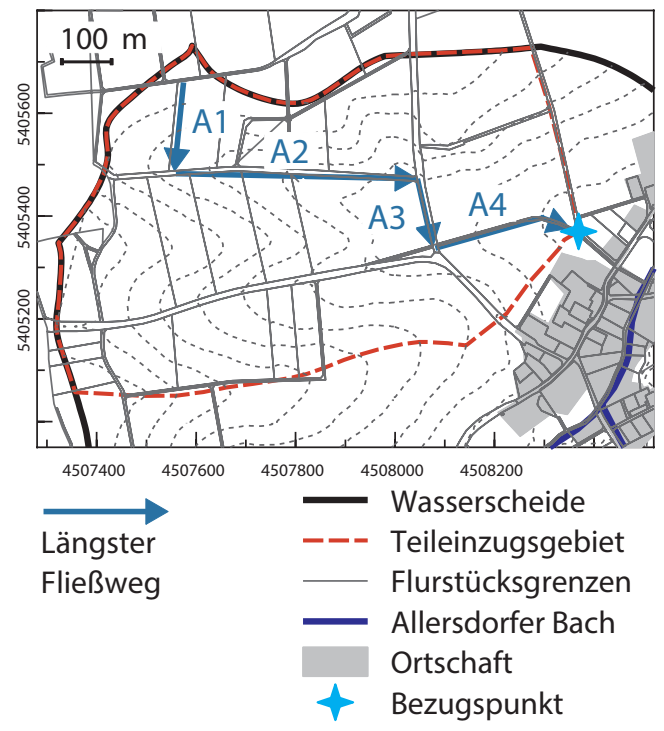

- Abb. 7.2 Darstellung des längsten Fließwegs ausgehend von einem Weg nahe der Wasserscheide bis zur Mündung in den Ortsbereich. Die einzelnen Pfeile (A1 bis A4) gliedern den Fließweg in Abschnitte mit ähnlichen hydraulischen Eigenschaften. (Geobasisdaten: Bayerische Vermessungsverwaltung)

\subsubsection{Abschätzung der Scheitelhöhe}

Zur Abschätzung der Scheitelhöhe wird das Dreiecksganglinienverfahren verwendet. Dabei bestimmen Abflusshöhe, Einzugsgebietsgröße, Scheitelanstiegs- und -ablaufzeit die Scheitelhöhe (vgl. - Abschn. 3.2.1). Die Einzugsgebietsgröße $\left(\mathrm{A}_{\mathrm{EZG}}\right)$ ergibt sich aus Gebietseigenschaften. Abflusshöhe $\left(\mathrm{N}_{\text {eff }}\right)$ und Scheitelanstiegszeit $\left(t_{p}\right)$ werden mit dem CN-Verfahren bzw. der Geschwindigkeitsmethode ermittelt, indem angenommen wird, dass die Scheitelanstiegszeit $\left(t_{\mathrm{p}}\right)$ der Konzentrationszeit $\left(t_{C}\right)$ des Gebietes entspricht. Die Ablaufzeit der Abflusswelle $\left(t_{\text {fal }}\right)$ hängt von den Retentionseigenschaften des Einzugsgebietes ab. Sie berechnet sich aus dem Produkt von Konzentrationszeit und einem Formfaktor $\mathrm{F}(-$ ) (hier $\mathrm{F}=1,5$ ) (vgl. - Tab. 3.2 und - Gl. 3.2). Sind alle erforderlichen Parameter und Größen bekannt, ergibt sich der Scheitelabfluss $\left(\mathrm{q}_{\mathrm{P}}\right)$ nach (vgl. $>\mathrm{Gl}$. 3.3):

$$
q_{P}=\frac{N_{e f f}}{0,5 \cdot\left(t_{c}+t_{f a l}\right) \cdot 0,06} \cdot A_{E Z G}
$$

\subsection{Ergebnisse}

\subsubsection{Abflussentstehung}

Die maximal mögliche Retention (S) wird nach $>$ Gl. 4.1 ermittelt. Für den CN-Wert von 80 ergibt sie sich zu:

$$
\begin{aligned}
S=254 \cdot\left(\frac{100}{C N}-1\right) & =254 \cdot\left(\frac{100}{80}-1\right) \\
& =63,5 \mathrm{~mm}
\end{aligned}
$$

Für die gewählte Niederschlagshöhe von $51 \mathrm{~mm}$ errechnet sich mit $>$ Gl. 4.2 ein abflusswirksamer Niederschlag $\mathrm{N}_{\text {eff }}$ von:

$$
\begin{aligned}
N_{\text {eff }} & =\frac{(N-0,2 \cdot S)^{2}}{N+0,8 \cdot S}=\frac{(51-0,2 \cdot 63,5)^{2}}{51+0,8 \cdot 63,5} \\
& =14,4 \mathrm{~mm}
\end{aligned}
$$

Aufgrund der Teileinzugsgebietsfläche von 47 ha ist bei dem gewählten Niederschlag ein Gesamtabflussvolumen zu erwarten in Höhe von $(\triangleright \mathrm{Gl} .2 .4)$ :

$$
Q=0,0144 \mathrm{~m} \cdot 470.000 \mathrm{~m}^{2}=6772 \mathrm{~m}^{3}
$$

\subsubsection{Konzentrationszeit}

\subsubsection{Bestimmung der Fließzeit von Schichtabfluss und flachem, konzentriertem Abfluss in Rinnen und Rillen}

Der erste Abschnitt A1 führt über eine Ackerfläche von der Wasserscheide nahezu senkrecht nach Süden bis zum ersten Graben. Es wird angenommen, dass der Oberflächenabfluss in diesem Abschnitt zunächst als Schichtabfluss auftritt, dessen Länge rechnerisch $\mathrm{zu}$ bestimmen ist, weil dies aus Luftbildern 


\begin{tabular}{|c|c|c|c|c|c|c|}
\hline $\begin{array}{l}\text { Fließpfad- } \\
\text { abschnitt }\end{array}$ & Abflusstyp & $\begin{array}{l}\text { Länge } \\
\text { (m) }\end{array}$ & $\begin{array}{l}\text { Gefälle } \\
(\%)\end{array}$ & $\begin{array}{l}\text { Rauheit } \\
\left(\mathrm{m}^{1 / 3} \mathrm{~s}^{-1}\right)\end{array}$ & $\begin{array}{l}\text { Sohlbreite } \\
\text { (m) }\end{array}$ & $\begin{array}{l}\text { Böschungs- } \\
\text { neigung }^{a}(\mathrm{~m} / \mathrm{m})\end{array}$ \\
\hline $\mathrm{A} 1$ & $\begin{array}{l}\text { Schichtabfluss und } \\
\text { Abfluss in Rinnen und } \\
\text { Rillen im Feld }\end{array}$ & 200 & 7,0 & 17 & - & - \\
\hline $\mathrm{A} 2$ & $\begin{array}{l}\text { Abfluss im trapez- } \\
\text { förmigen Graben }\end{array}$ & 470 & 2,4 & 35 & 0,35 & 1 \\
\hline A3 & $\begin{array}{l}\text { Abfluss im trapez- } \\
\text { förmigen Graben }\end{array}$ & 180 & 3,0 & 35 & 0,35 & 1 \\
\hline A4 & $\begin{array}{l}\text { Abfluss im trapez- } \\
\text { förmigen Graben }\end{array}$ & 300 & 2,2 & 35 & 0,7 & 0,6 \\
\hline
\end{tabular}

und auch im Gelände, außer bei einem entsprechenden Starkregen, nicht zu ermitteln ist. Danach fließt der Abfluss immer noch im Feld in flachen Rinnen und Rillen bis zum Hangfuß. Da die Hänge bei A1 relativ kurz, gerade und ohne nennenswerte Einmuldung ausgebildet sind, ist eine Konzentration des Abflusses in Hangmulden nicht zu erwarten.

Die Länge des Schichtabflusspfades wird mithilfe von > Gl. $8.1 \mathrm{im}$ Anhang anhand der Rauheit der Bodenoberfläche abgeschätzt. Für einen Acker mit spärlicher Bedeckung (Bedeckung $<5 \%$ ), weist - Tab. 8.2 eine Rauheit von $\mathrm{k}=17\left(\mathrm{~m}^{1 / 3} \mathrm{~s}^{-1}\right)$ aus. Demnach ergibt sich für den Schichtabfluss eine Fließpfadlänge von:

$$
l_{s}=\frac{200}{\sqrt{k}}=\frac{200}{\sqrt{17}}=49 \mathrm{~m}
$$

Aus der Differenz der Gesamtlänge des Abschnitts A1 und der Fließstrecke des Schichtabflusses ergibt sich die mittlere Länge des Rinnen- und Rillenabflusses:

$$
l_{R}=200-49=151 \mathrm{~m}
$$

Auf diese beiden Fließstrecken wird nun die GMS-Gleichung ( $>$ Gl.5.5) angewendet. Eingangsgrößen für den Schichtabfluss sind ein Gefälle J von 7 \% (bestimmt aus der topographischen Karte), die bereits festgelegte
Rauheit der Oberfläche $\mathrm{k}$ von $17 \mathrm{~m}^{1 / 3} \mathrm{~s}^{-1}$ und der hydraulische Radius R. Letzterer wird nach - Anhang 8.2.1 vereinfachend für einen schichtförmigen Abfluss mit $2 \mathrm{~mm}$ geschätzt. Als Fließgeschwindigkeit ergibt sich:

$$
\begin{aligned}
v & =R^{\frac{2}{3}} \cdot J^{\frac{1}{2}} \cdot k \\
& =0,002^{\frac{2}{3}} \cdot 0,07^{\frac{1}{2}} \cdot 17 \\
& =0,07 \mathrm{~m} \mathrm{~s}^{-1} \\
& =7 \mathrm{~cm} \mathrm{~s}^{-1}
\end{aligned}
$$

Dies ist ein typischer Wert für den Schichtabfluss. Wird diese Geschwindigkeit durch die Länge des Schichtabflusspfades dividiert, ergibt sich nach $>$ Gl. 5.7 eine Fließzeit von

$$
t_{S}=\frac{49}{60 \cdot 0,07}=11,3 \mathrm{~min}
$$

Für den Abfluss in Rinnen und Rillen wird ebenfalls von einem Gefälle J von $7 \%$ ausgegangen. Durch die kastenförmige Ausspülung der Fließpfade liegt die Rauheit analog - Tab. $8.2 \mathrm{im}$ Anhang bei $\mathrm{k}=25 \mathrm{~m}^{1 / 3}$ $\mathrm{s}^{-1}$. Der hydraulische Radius $\mathrm{R}$ wird pauschal mit 0,04 $\mathrm{m}$ angesetzt ( $\downarrow$ Anhang 8.2.2). Damit ergibt sich die Fließgeschwindigkeit zu:

$$
v=0,04^{\frac{2}{3}} \cdot 0,07^{\frac{1}{2}} \cdot 25=0,8 \mathrm{~m} \mathrm{~s}^{-1}
$$


Durch die Konzentration des Abflusses in flachen Rillen und die geringere Rauheit fließt der Abfluss hier bereits fast 10-mal schneller als der Schichtabfluss. Würde die Konzentration des Abflusses in Rillen durch eine höhere Rauheit, z. B. durch eine Mulchschicht, hinausgezögert, würde die Gesamtzeit, die der Abfluss sich im Fließabschnitt A1 bewegt, deutlich verlängert. Wird die Länge der Rinnen und Rillen durch die Fließgeschwindigkeit dividiert, resultiert eine mittlere Fließzeit von:

$$
t_{R}=\frac{151}{60 \cdot 0,8}=3,3 \mathrm{~min}
$$

\subsubsection{Bestimmung der Fließzeit in den Wegseitengräben}

Erreicht der Oberflächenabfluss am Hangfuß das Ende von Abschnitt A1, tritt er in den Wegseitengraben über. Letzterer verläuft zunächst nach Osten bis zur T-Kreuzung der Straße (Abschnitt A2). Dort führt eine Verrohrung in einen kurzen, in NordSüd-Richtung verlaufenden Graben (Abschnitt A3), der am südlichsten Punkt an den Sammelgraben (Abschnitt A4) angeschlossen ist (• Abb. 7.2).

Die Fließzeit in den Grabenabschnitten wird indirekt geschätzt, da für die GMSGleichung die Fließtiefe erforderlich ist. Daher muss zunächst die Fließtiefe näherungsweise berechnet werden. Dazu werden die Gleichungen aus - Abb. 8.4 zur Bestimmung von Gerinnequerschnittsfläche (A) und hydraulischem Radius (R) von Trapezen,

$$
A=\frac{1}{2}(B+b) \cdot h=\frac{1}{2}(2 b+2 m \cdot h) \cdot h
$$

und

$$
R=\frac{(b+m \cdot h) \cdot h}{b+2 h \sqrt{1+m^{2}}}
$$

und die GMS-Gleichung (vgl. > Gl. 5.5),

$$
v=R^{\frac{2}{3}} \cdot J^{\frac{1}{2}} \cdot k
$$

sowie die allgemeine Fließgleichung benötigt (vgl. $>\mathrm{Gl} .5 .1)$

$$
q=v \cdot A
$$

Dabei sind B, b und h Wasserspiegelbreite, Sohlbreite und Fließtiefe des trapezförmigen Grabens in $\mathrm{m}$. Die Böschungsneigung (m) ist dimensionslos, $\mathrm{R}$ ist der hydraulische Radius in $\mathrm{m}$, J das Gefälle (dimensionslos) und $\mathrm{k}$ der Rauheitsbeiwert in $\mathrm{m}^{1 / 3} \mathrm{~s}^{-1}$. In der letzten Formel steht v für die Fließgeschwindigkeit in $\mathrm{m} \mathrm{s}^{-1}$, die Querschnittsfläche hat die Einheit $\mathrm{m}^{2}$.

Werden die Gleichungen aus - Abb. 8.4, 5.5 und 5.1 ineinander eingesetzt und durch die Einzugsgebietsfläche dividiert, ergibt sich die Abflussspende $\left(\mathrm{q}_{\mathrm{s}}\right)$ in Abhängigkeit von der Fließtiefe (h) nach:

$$
\begin{aligned}
q_{s}(h)= & \left(\frac{(b+m \cdot h) \cdot h}{b+2 h \sqrt{1+m^{2}}}\right)^{\frac{2}{3}} \cdot J^{\frac{1}{2}} \cdot k \\
& \cdot \frac{1}{2}(2 b+2 m \cdot h) \cdot h \cdot \frac{1}{A_{E Z G}}
\end{aligned}
$$

Die Gleichung kann nun gelöst werden, indem die Werte eingesetzt und die Fließtiefe $\mathrm{h}$ schrittweises (iterativ) verändert wird, bis das resultierende $\mathrm{q}_{\mathrm{s}}$ etwa der erforderlichen Abflusshöhe von $14,4 \mathrm{~mm} \mathrm{~h}^{-1}$ entspricht. Dazu wird der Abfluss im Graben unter Berücksichtigung der Einzugsgebietsfläche $\left(\mathrm{A}_{\mathrm{EZG}}\right)$ am Übergang von $\mathrm{A} 2$ nach $\mathrm{A} 3$ $\left(A_{E Z G}=10\right.$ ha bzw. $\left.100.000 \mathrm{~m}^{2}\right)$ berechnet (stationäre Bedingungen). Für das gewählte Regenereignis ergibt sich eine Fließtiefe im ersten, $470 \mathrm{~m}$ langen Grabenabschnitt von rund $34 \mathrm{~cm}$ :

$$
\begin{aligned}
q_{s}= & \left(\frac{(0,35+1 \cdot 0,34) \cdot 0,34}{0,35+2 \cdot 0,34 \sqrt{1+1^{2}}}\right)^{\frac{2}{3}} \cdot 0,024^{\frac{1}{2}} \cdot 35 \\
& \cdot \frac{1}{2}(2 \cdot 0,35+2 \cdot 1 \cdot 0,34) \cdot 0,34 \cdot \frac{1000 \cdot 3600}{100.000} \\
\approx & 14,3 \mathrm{~mm} \mathrm{~h}^{-1}
\end{aligned}
$$

Ist die Fließtiefe bekannt, lässt sich die Fließgeschwindigkeit abschätzen: 


$$
\begin{aligned}
v & =R^{\frac{2}{3}} \cdot J^{\frac{1}{2}} \cdot k=\left(\frac{(b+m \cdot h) \cdot h}{b+2 h \sqrt{1+m^{2}}}\right)^{\frac{2}{3}} \cdot J^{\frac{1}{2}} \cdot k \\
& =\left(\frac{(0,35+0,34) \cdot 0,34}{0,35+2 \cdot 0,34 \sqrt{1+1}}\right)^{\frac{2}{3}} \cdot 0,024^{\frac{1}{2}} \cdot 35 \\
& =1,7 \mathrm{~m} \mathrm{~s}^{-1}
\end{aligned}
$$

Durch Division von Fließstrecke und -geschwindigkeit ergibt sich die Fließzeit im ersten Grabenabschnitt zu:

$$
t=\frac{l}{v}=\frac{470}{1,7 \cdot 60}=4,6 \min
$$

Für den in Nord-Süd-Richtung verlaufenden Grabenabschnitt A3 und den Teil des Sammelgrabens bis zum Beginn der Ortschaft (A4) werden die Rechenschritte ana$\log$ wiederholt. Die Einzugsgebietsfläche am Übergang von A3 zu A4 beträgt etwa 12 ha. In A3 ergibt sich eine ähnliche mittlere Fließtiefe von rund $0,35 \mathrm{~m}$, da sich die entgegengesetzt gerichteten Wirkungen der größeren Einzugsgebietsfläche und des höheren Gefälles kompensieren:

$$
\begin{aligned}
q_{s}= & \left(\frac{(0,35+1 \cdot 0,35) \cdot 0,35}{0,35+2 \cdot 0,35 \sqrt{1+1^{2}}}\right)^{\frac{2}{3}} \cdot 0,03^{\frac{1}{2}} \cdot 35 \\
& \cdot \frac{1}{2}(2 \cdot 0,35+2 \cdot 0,35) \cdot 0,35 \cdot \frac{1000 \cdot 3600}{120.000} \\
\approx & 14,4 \mathrm{~mm} \mathrm{~h}^{-1}
\end{aligned}
$$

Gegenüber dem ersten Grabenabschnitt ist die mittlere Fließgeschwindigkeit in A3 durch das höhere Gefälle geringfügig höher:

$$
\begin{aligned}
v & =R^{\frac{2}{3}} \cdot J^{\frac{1}{2}} \cdot k \\
& =\left(\frac{(b+m \cdot h) \cdot h}{b+2 h \sqrt{1+m^{2}}}\right)^{\frac{2}{3}} \cdot J^{\frac{1}{2}} \cdot k \\
& =\left(\frac{(0,35+1 \cdot 0,35) \cdot 0,35}{0,35+2 \cdot 0,35 \sqrt{1+1}}\right)^{\frac{2}{3}} \cdot 0,03^{\frac{1}{2}} \cdot 35 \\
& =2,0 \mathrm{~m} \mathrm{~s}^{-1}
\end{aligned}
$$

Daraus errechnet sich die mittlere Fließzeit zu:

$$
t=\frac{l}{v}=\frac{180}{2,0 \cdot 60}=1,5 \mathrm{~min}
$$

Der Sammelgraben (A4) entwässert nun nahezu das komplette Teileinzugsgebiet von 47 ha bis zum Bezugspunkt. Die Bestimmung der Fließzeit erfolgt analog zu dem Vorgehen bei A2 und A3 mit den in - Tab. 7.2 genannten Größen. Um die gewünschte Abflusshöhe $\mathrm{zu}$ erreichen, ist eine Fließtiefe von $68 \mathrm{~cm}$ erforderlich.

$$
\begin{aligned}
q_{s}= & \left(\frac{(0,7+0,6 \cdot 0,68) \cdot 0,68}{0,7+2 \cdot 0,68 \sqrt{1+0.6^{2}}}\right)^{\frac{2}{3}} \cdot 0,022^{\frac{1}{2}} \cdot 35 \\
& \cdot \frac{1}{2}(2 \cdot 0,7+2 \cdot 0,6 \cdot 0,68) \cdot 0,68 \cdot \frac{1000 \cdot 3600}{470.000} \\
\approx & 14,3 \mathrm{~mm} \mathrm{~h}^{-1}
\end{aligned}
$$

Nach den gleichen Prinzipien wie oben ergeben sich die mittlere Fließgeschwindigkeit $\mathrm{zu}$

$$
\begin{aligned}
v & =R^{\frac{2}{3}} \cdot J^{\frac{1}{2}} \cdot k=\left(\frac{(b+m \cdot h) \cdot h}{b+2 h \sqrt{1+m^{2}}}\right)^{\frac{2}{3}} \cdot J^{\frac{1}{2}} \cdot k \\
& =\left(\frac{(0,7+0,6 \cdot 0,68) \cdot 0,68}{0,7+2 \cdot 0,68 \sqrt{1+0,6^{2}}}\right)^{\frac{2}{3}} \cdot 0,022^{\frac{1}{2}} \cdot 35 \\
& =2,5 \mathrm{~m} \mathrm{~s}^{-1}
\end{aligned}
$$

und die mittlere Fließzeit zu:

$$
t=\frac{l}{v}=\frac{300}{2,5 \cdot 60}=2,0 \mathrm{~min}
$$

Entsprechend der einzelnen Abschnitte des Fließpfads ergibt sich von der Wasserscheide bis zum Erreichen des Bezugspunktes im Sammelgraben am Ortseingang von Birnbach für den Oberflächenabfluss eine mittlere Konzentrationszeit $\left(\mathrm{t}_{\mathrm{C}}\right)$ von:

$$
t_{C}=11,3+3,3+4,6+1,5+2,0 \approx 23 \mathrm{~min}
$$

Obwohl der Fließweg, auf dem der Abfluss schichtförmig fließt, nur $49 \mathrm{~m}$ oder $5 \%$ der gesamten Fließstrecke bis zum Ende von A3 ausmacht, verweilt hier der Abfluss $50 \%$ der gesamten Abflussdauer. Das heißt, dass in diesem Fall die Dämpfung der Hochwasserwelle auf den ersten $49 \mathrm{~m}$ genauso stark ist wie auf den folgenden $1100 \mathrm{~m}$. Dies zeigt die fatale Wirkung der Bündelung des Abflusses bzw., wie nützlich es ist, wenn der Abfluss 
möglichst lange schichtförmig fließt. Das ist der wesentliche Vorteil von Grünland oder einer Mulchdirektsaat mit einer durchgehenden Bodenbedeckung $\geq 30 \%$, wo das Einschneiden in Rillen und Rinnen normalerweise nicht erfolgt. Für Grünland hätte man über die ganzen $200 \mathrm{~m}$ von Abschnitt A1 schichtförmiges Fließen mit einer entsprechend starken Dämpfung der Hochwasserwelle annehmen können.

Selbst im unteren Teilstück von A1, in dem der Abfluss bereits in Rillen und Rinnen fließt, ist die Fließgeschwindigkeit nur halb so hoch wie in den Gräben, obwohl die Neigung des Feldes mehr als doppelt so stark ist, wie die der Gräben. Dies zeigt die starke Beschleunigung, die ein Abfluss durch Einengung in ein glattes Gerinne erfährt. Je länger der Abfluss breit fließen kann und je breiter er fließt, nachdem er gebündelt wurde, umso länger und stärker gedämpft ist er unterwegs. Der moderne Wegeund Straßenbau kann also höchst problematisch für den Landschaftswasserhaushalt sein, weil dadurch oft ein sekundäres Gewässernetz entsteht, dass durch Oberflächen- und Zwischenabfluss bei starken Niederschlägen aktiviert wird (• Abb. 7.3). Es leitet Abfluss und Abtrag aus den Hanglagen, wo der Abtrag mindestens

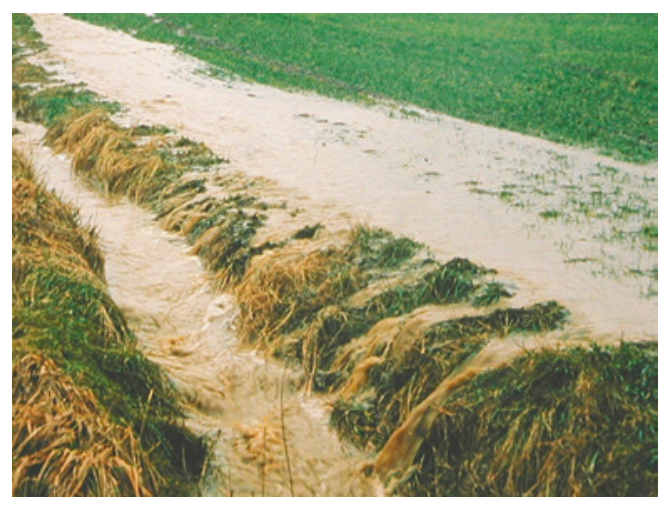

- Abb. 7.3 Selbst kleine Entwässerungsgräben (hier der Wegseitengraben eines Feldwegs) beschleunigen den Abfluss stark, wie deutlich am unterschiedlichen Strömungsmuster im Graben links und auf dem Feld rechts zu erkennen ist um Faktor 10 bis 100 höher ist als in den Tallagen, direkt und ungepuffert in das primäre Fließgewässernetz. Daher ist es wenig verwunderlich, dass Zusammenhänge zwischen Landnutzungseffekten, Feinsedimentdeposition und den aquatischen Lebensgemeinschaften nachgewiesen wurden $[2,3]$.

\subsubsection{Scheitelabflussrate bei Starkregen}

Anhand der Oberflächenabflusshöhe $\mathrm{N}_{\text {eff }}$ in $\mathrm{mm}$, der Scheitelanstiegszeit $t_{\mathrm{p}}$ in $\min$ (die $t_{C}$ gleichgesetzt wird), der Zeit des fallenden Wellenastes $t_{\text {fal }}$ in min und der Gesamtgröße des Teileinzugsgebietes $A_{E Z G}$ in $\mathrm{km}^{2}$ lässt sich über das Dreiecksganglinienverfahren der Abflussscheitel $\mathrm{q}_{\mathrm{P}}$ in $1 \mathrm{~s}^{-1}$ für das gewählte Starkregenszenario abschätzen. Wird vereinfachend davon ausgegangen, dass die Teilgebiete $\mathrm{B}$ und C identische Konzentrationszeiten wie Teilgebiet A aufweisen, überlagern sich die Abflusswellen der einzelnen Teilflächen beim Eintritt in den Sammelgraben A4 linear (ungünstigster Fall). Für diesen Fall bestimmt die Flächengröße die Scheitelhöhe und bei einem 100-jährlichen Starkregen muss mit einem Spitzenabfluss von knapp $4000 \mathrm{l} \mathrm{s}^{-1}$ gerechnet werden.

$$
\begin{aligned}
q_{P} & =\frac{N_{e f f}}{0,5 \cdot\left(t_{c}+t_{f a l}\right) \cdot 0,06} \cdot A \\
& =\frac{14,4}{0,5 \cdot(23+1,5 \cdot 23) \cdot 0,06} \cdot 0,47 \\
& =39701 \mathrm{~s}^{-1}
\end{aligned}
$$

\subsubsection{Einfluss der Grabengestaltung auf Abflusskonzentration und Scheitelabflussrate}

Um Möglichkeiten der Grabengestaltung zur Minderung des Abflussscheitels einzuschätzen, wird angenommen, dass die bestehenden Wegseitengräben aufgeweitet und abgeflacht werden (Sohlbreite $b=1,5 \mathrm{~m}$ 
für A2 und A3 bzw. 2 m für A4, Böschungsneigung $m$ einheitlich 2,5) und sich durch den Verzicht auf eine regelmäßige Mahd ein dichter, hydraulisch rauer Bewuchs einstellt (Rauheitsbeiwert $\mathrm{k}$ von $20 \mathrm{~m}^{1 / 3} \mathrm{~s}^{-1}$ ). Werden die dargestellten Rechenschritte für diese Randbedingungen wiederholt, erhöht sich die Fließzeit gegenüber dem aktuellen Zustand um $30 \%$ von rund 23 auf $30 \mathrm{~min}$. - Tab. 7.3 fasst die Berechnungsergebnisse für einen Abfluss von 14,4 mm zusammen.

Wird nun die längere Fließzeit von rund 30 min verwendet, um den Abflussscheitel am Bezugspunkt $\mathrm{zu}$ schätzen, errechnet sich aus dem Dreiecksganglinienverfahren eine um $25 \%$ reduzierte Scheitelhöhe von rund $30001 \mathrm{~s}^{-1}$ :

$$
\begin{aligned}
q_{P} & =\frac{N_{e f f}}{0,5 \cdot\left(t_{c}+t_{f a l}\right) \cdot 0,06} \cdot A \\
& =\frac{14,4}{0,5 \cdot(29,5+1,5 \cdot 29,5) \cdot 0,06} \cdot 0,47 \\
& =30551 \mathrm{~s}^{-1}
\end{aligned}
$$

\subsubsection{Wirkung einer flächendeckenden Mulchdirektsaat}

Würde dagegen die Bewirtschaftungsrichtung um $90^{\circ}$ gedreht, was z. B. im Teilgebiet A einfach möglich wäre, und würde flächendeckend auf Mulchdirektsaat umgestellt, könnte für das flächenhafte Fließen statt einem Rauheitsbeiwert von $17 \mathrm{~m}^{1 / 3} \mathrm{~s}^{-1}$ einer von $8 \mathrm{~m}^{1 / 3} \mathrm{~s}^{-1}$ angenommen werden (vgl. - Tab. 8.2). Dieser niedrige Rauheitsbeiwert kommt daher, dass bei Mulchdirektsaat eine raue, abflussbremsende Bodenoberfläche selbst zehn Monate nach der Bodenbearbeitung noch erhalten bleibt (• Abb. 7.4), weil die abfrierende Zwischenfrucht in ein sehr raues Saatbett gesät werden kann. Die sich im August rasch entwickelnde Zwischenfrucht schützt diese Rauheit vor der einebnenden Wirkung der Witterung, sodass sie selbst im Mai, wenn der Mais keimt, noch zum großen Teil vorhanden ist. Zusätzlich liefern zunächst die wachsende Zwischenfrucht und dann nach dem Winter ihre abgefrorenen Reste eine dichte, abflussbremsende Mulchdecke. Durch die noch aufrechtstehenden, verwurzelten Stängel ist die Mulchdecke hervorragend gegen Abschwemmung geschützt. Gleichzeitig erfüllt die Mulchdirektsaat auch die konträren Forderungen, die an ein optimales Saatbett gestellt werden und die normalerweise nur durch aufwendige Bodenbearbeitung gleichzeitig realisiert werden können: Der für die Keimung notwendige kapillare Anschluss des Saatkorns ist durch die zehnmonatige Bodensetzung ohne weitere Bearbeitung ideal. Im konventionellen Saatbett muss dies dagegen durch eine technische Bodenverdichtung erzeugt werden. Gleichzeitig verringert die Mulchschicht die Bodenverdunstung und damit einen unproduktiven Wasserverlust. Im konventionellen Saatbett erreicht man dies durch eine wenige Zentimeter dicke, durch intensives Zerschlagen von Bodenaggregaten erzeugte Schicht, die auf der Verdichtungszone aufliegt. Diese Schicht aus Feinbröckeln ist hydraulisch glatt und wird rasch durch Regen weiter geglättet, da die Bröckel durch die intensive Bearbeitung instabil sind [4]. Sobald Oberflächenabfluss auftritt, kann Feinmaterial von dieser auf der Verdichtungszone aufliegenden Feinbröckelschicht leicht abgeschwemmt werden. Die Erosionsrate ist dann hoch. Bei Mulchdirektsaat verhindert dagegen die Mulchschicht ein Verschlämmen und hält die Infiltrationsrate hoch. Wird die Infiltration trotzdem überschritten, baut sich eine dicke Wasserschicht auf, die zusätzlich zur Mulchschicht ein Auftreffen der Regentropfen auf der Bodenoberfläche und die Produktion von abschwemmbarem Feinmaterial reduziert $[5,6]$.

Durch diese Maßnahme würde, weil das Wasser nun langsamer fließt und sich dadurch auch erst später in Rillen und Rinnen bündelt, die Fließzeit im Feld auf 26 min steigen (rund $80 \%$ mehr) und entsprechend der Hochwasserscheitel auf rund $26001 \mathrm{~s}^{-1} \quad(34 \%$ weniger als im aktuellen Zustand) sinken. Weil dadurch aber auch die Infiltration verbessert wird (nach > Gl. 4.8 und 4.9 kann für Mulchdirektsaat und konturparallelen 
- Tab. 7.3 Zusammenfassung der Berechnungen für die Grabenabschnitte A2, A3 und A4 bei einer Abflussrate von $14,4 \mathrm{~mm} \mathrm{~h}^{-1}$ eines 100 -jährlichen Regens. „Aktuell” gilt für die gegenwärtige Grabengestalt, das Szenario „Aufgeweitet + Bewuchs“ gilt für flachere und hydraulisch raue Verhältnisse

\begin{tabular}{|c|c|c|c|}
\hline & Parameter & Aktuell & Aufgeweitet \& Bewuchs \\
\hline \multirow[t]{10}{*}{ A2 } & Sohlbreite b (m) & 0,35 & 1,5 \\
\hline & Böschungsneigung $\mathrm{m}\left(\mathrm{m} \mathrm{m}^{-1}\right)$ & 1,0 & 2,5 \\
\hline & Fließtiefe d (m) & 0,34 & 0,21 \\
\hline & Wasserspiegelbreite B (m) & 1,0 & 2,6 \\
\hline & Grabenöffnungsweite (m) & 1,8 & 5,0 \\
\hline & Durchflossene Querschnittsfläche A (m²) & 0,23 & 0,43 \\
\hline & Hydraulischer Radius R (m) & 0,18 & 0,16 \\
\hline & Fließgeschwindigkeit v $\left(\mathrm{m} \mathrm{s}^{-1}\right)$ & 1,7 & 0,9 \\
\hline & Mittlere Abflussrate $Q$ bei $A E Z G=10$ ha $\left(I ~^{-1}\right)$ & 400 & 400 \\
\hline & Fließzeit Abschnitt A2 (min) & 4,6 & 8,5 \\
\hline \multirow[t]{10}{*}{ A3 } & Sohlbreite b (m) & 0,35 & 1,5 \\
\hline & Böschungsneigung $\mathrm{m}\left(\mathrm{m}^{-1}\right)$ & 1,0 & 2,5 \\
\hline & Fließtiefe $d(m)$ & 0,35 & 0,22 \\
\hline & Wasserspiegelbreite B (m) & 1,1 & 2,6 \\
\hline & Grabenöffnungsweite (m) & 1,8 & 5,0 \\
\hline & Durchflossene Querschnittsfläche A (m²) & 0,25 & 0,45 \\
\hline & Hydraulischer Radius R (m) & 0,18 & 0,17 \\
\hline & Fließgeschwindigkeit $v\left(\mathrm{~m} \mathrm{~s}^{-1}\right)$ & 2,0 & 1,1 \\
\hline & Mittlere Abflussrate $Q$ bei $A_{E Z G}=12$ ha $\left(\mid \mathrm{s}^{-1}\right)$ & 480 & 480 \\
\hline & Fließzeit Abschn. A3 (min) & 1,5 & 2,8 \\
\hline \multirow[t]{10}{*}{ A4 } & Sohlbreite b (m) & 0,70 & 2 \\
\hline & Böschungsneigung $\mathrm{m}\left(\mathrm{m}^{-1}\right)$ & 0,60 & 2,5 \\
\hline & Fließtiefe d (m) & 0,68 & 44 \\
\hline & Wasserspiegelbreite B (m) & 1,5 & 4,2 \\
\hline & Grabenöffnungsweite (m) & 1,5 & 5,5 \\
\hline & Durchflossene Querschnittsfläche A $\left(\mathrm{m}^{2}\right)$ & 0,76 & 1,4 \\
\hline & Hydraulischer Radius R (m) & 0,33 & 0,31 \\
\hline & Fließgeschwindigkeit v $\left(\mathrm{m} \mathrm{s}^{-1}\right)$ & 2,5 & 1,4 \\
\hline & Mittlere Abflussrate $Q$ bei $A_{E Z G}=47$ ha $\left(\mathrm{s}^{-1}\right)$ & 1880 & 1880 \\
\hline & Fließzeit Abschn. A4 (min) & 2,0 & 3,7 \\
\hline A1 & Fließzeit im Feld (schichtförmig + Rinnen + Rillen) (min) & 14,6 & 14,6 \\
\hline \multirow[t]{2}{*}{ A1-A4 } & Summe Fließzeit (min) & 22,7 & 29,5 \\
\hline & Summe relativ $(\%)$ & 100 & 130 \\
\hline
\end{tabular}




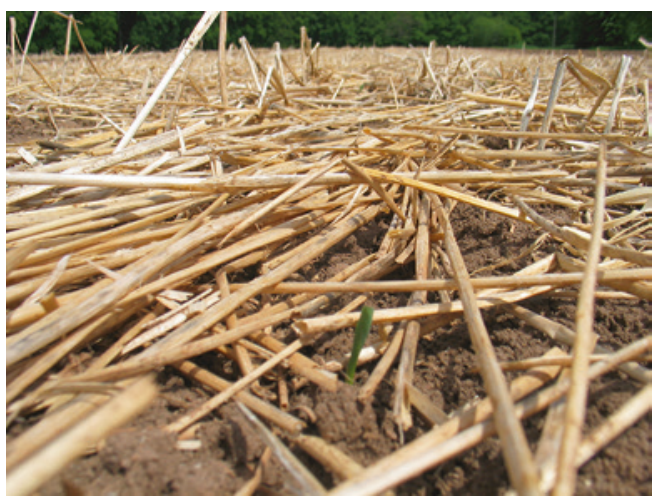

- Abb. 7.4 Raue, abflussbremsende Bodenoberfläche selbst zehn Monate nach der Bodenbearbeitung bei Mulchdirektsaat. (Bildquelle: Robert Brandhuber)

Anbau ein CN-Wert von 69 angesetzt werden), würden bei diesem Szenario nur $5,3 \mathrm{~mm}$ anstelle von $14,4 \mathrm{~mm}$ abfließen (ca. $60 \%$ weniger; diese enorme Wirkung wurde auch in Beregnungsversuchen nachgewiesen [7]). Dadurch würde das Wellenvolumen und damit der Abflussscheitel um weitere $40 \%$ auf rund $1000 \mathrm{l} \mathrm{s}^{-1}$ gesenkt. In der Folge führt das gesamte Entwässerungssystem weniger Wasser. Dadurch wiederum fließt auch in den folgenden Abschnitten, den Rillen und Rinnen und in A2 bis A4, der Abfluss aufgrund der geringen Schichtdicke langsamer, was den Abflussscheitel weiter senkt, und zwar um $5 \%$. Als willkommener "Nebeneffekt" wird gleichzeitig auch der Bodenabtrag mehr als halbiert, was die Bodenfruchtbarkeit erhöht und die Grabenräumungskosten senkt. Weil die Gräben nun sehr viel weniger verschlammen, macht es erst wirklich Sinn, die Fließgeschwindigkeit in den Gräben durch eine Umgestaltung zu vermindern. Kombiniert man also die veränderte Flächenbewirtschaftung mit der oben dargestellten, veränderten Grabengestaltung, kann der Hochwasserscheitel eines kurzen, schauerartigen Niederschlags im betrachteten Gebiet um bis $\mathrm{zu} \quad 80 \%$ reduziert werden

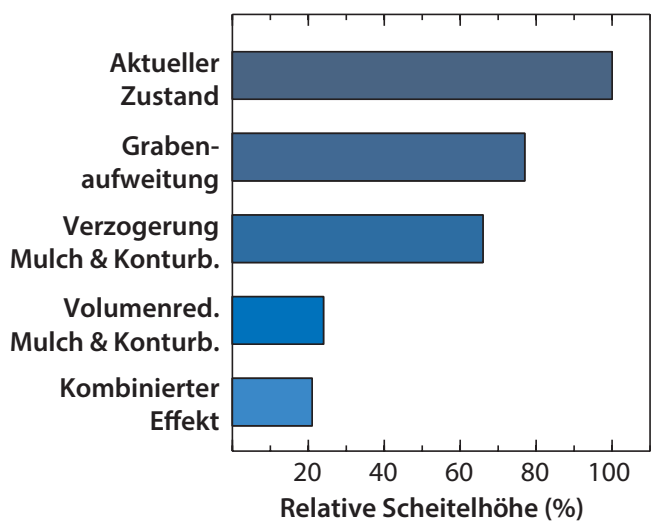

- Abb. 7.5 Exemplarische Darstellung der potenziellen Reduktion des Abflussscheitels durch Maßnahmen zur Volumenreduktion und Abflussverzögerung in der Flur bei kurzen, schauerartigen Starkregenereignissen in einem Teileinzugsgebiet des Allersdorfer Baches

(• Abb. 7.5). Das Überflutungsrisiko für die Gemeinde sollte damit deutlich reduziert werden können - oder umgekehrt: Die wiederkehrenden Hochwasserschäden sind durch den Menschen verursacht.

Die Effekte der einzelnen Maßnahmen sind in - Abb. 7.5 gegenübergestellt. Sie gelten nur für das betrachtete Gebiet und die genannten Randbedingungen. Eine exakte Trennung und Quantifizierung der einzelnen Effekte ist streng genommen nicht möglich, da sie ineinandergreifen. Die Auswertung soll nur den Beitrag der einzelnen Maßnahmen zur Gesamtwirkung exemplarischen verdeutlichen.

\subsection{Zusammenfassung}

In der Ortschaft Birnbach treten immer wieder kleinere Überschwemmungen auf. Ursache sind die Kessellage der Ortschaft, die flächendeckend landwirtschaftliche Bodennutzung und die durch Gräben künstlich beschleunigte Abflusskonzentration im Einzugsgebiet. Die Gräben sammeln und bündeln 
den bei Starkregen anfallenden Oberflächenabfluss und leiten ihn direkt in und durch Teilbereiche der Ortschaft.

Um den Einfluss der Gräben (und der Flächennutzung) auf die Abflusssituation einzuschätzen, wurden exemplarisch potenzielle Spitzenabflüsse und Fließzeiten des Oberflächenabflusses in einem Teileinzugsgebiet für einen etwa 100-jährlichen Starkregen ermittelt. Dabei wurde der aktuelle Zustand des Gebietes mit einem Szenario verglichen, bei dem die vorhandenen Wegseitengräben stark aufgeweitet und bewachsen, d. h. hydraulisch rau angelegt sind. Der Vergleich ergab, dass sich durch eine (durchgehende) Veränderung der Grabengestalt die Abflusskonzentration in dem ausgewählten Teileinzugsgebiet um rund $30 \%$ verzögern und der Scheitel der Abflusswelle um $25 \%$ reduzieren ließe. Bei einer weiterreichenden Veränderung (zusätzlich flächendeckende Umstellung auf Mulchdirektsaat und hangparallele Bewirtschaftung) wäre eine Abflussminderung um $50 \%$ und mehr gegenüber der aktuellen Situation zu erwarten und eine Minderung des Hochwasserscheitels um $80 \%$. Eine weitere Reduktion wäre mit überschaubarem Aufwand möglich durch gezielte Heterogenisierung des Anbaus (z. B. Streifenanbau) oder durch die Anlage von kleinen Retentionsbecken (besonders entlang der Fließstrecke A2 und am Übergang von A3 zu A4).

\section{Literatur}

1. Brandhuber $R$, Treisch $M$, Fischer $F$ et al (2017) Starkregen, Bodenerosion, Sturzfluten. Schriftenr Bayer Landesanst Landwirtsch 2: Freising

2. Bierschenk AM, Mueller M, Pander J, Geist J (2019) Impact of catchment land use on fish community composition in the headwater areas of Elbe, Danube and Main. Sci Total Environ 652:66-74

3. Knott J, Mueller M, Pander J, Geist J (2019) Effectiveness of catchment erosion protection measures and scale-dependent response of stream biota. Hydrobiologia 830:77-92

4. Auerswald K (1993) Infuence of initial moisture and time since tillage on surface structure breakdown and erosion of a loessial soil. Catena Suppl 24:93-101

5. Palmer RS (1964) The influence of a thin water layer on waterdrop impact forces. IAHS Publ 65:141-148

6. Poesen J, Savat J (1981) Detachment and transportation of loose sediments by raindrop splash Part II: Detachability and transportability measurements. Catena 8:19-41

7. Kainz M (1989) Runoff, erosion and sugar beet yields in conventional and mulched cultivation. Soil Technol Ser 1:103-114 
Open Access Dieses Kapitel wird unter der Creative Commons Namensnennung 4.0 International Lizenz ( $\$$ http://creativecommons.org/licenses/by/4.0/deed.de) veröffentlicht, welche die Nutzung, Vervielfältigung, Bearbeitung, Verbreitung und Wiedergabe in jeglichem Medium und Format erlaubt, sofern Sie den/ die ursprünglichen Autor(en) und die Quelle ordnungsgemäß nennen, einen Link zur Creative Commons Lizenz beifügen und angeben, ob Änderungen vorgenommen wurden.

Die in diesem Kapitel enthaltenen Bilder und sonstiges Drittmaterial unterliegen ebenfalls der genannten Creative Commons Lizenz, sofern sich aus der Abbildungslegende nichts anderes ergibt. Sofern das betreffende Material nicht unter der genannten Creative Commons Lizenz steht und die betreffende Handlung nicht nach gesetzlichen Vorschriften erlaubt ist, ist für die oben aufgeführten Weiterverwendungen des Materials die Einwilligung des jeweiligen Rechteinhabers einzuholen. 